Gynäkologe 2009 • 42:571-572

DOI 10.1007/s00129-009-2416-9

๑) Springer Medizin Verlag 2009

\author{
L. Beck ${ }^{1} \cdot$ J. Baltzer ${ }^{2}$ \\ ${ }^{1}$ Frauenklinik, Heinrich-Heine-Universität, Düsseldorf \\ 2 Krefeld
}

\title{
Verhalten in kritischen Rechtssituationen
}

Umfangreiche Tätigkeit in Klinik und Gutachterkommission hat die Bedeutung der Fragen erkennen lassen, die unter der Überschrift „Verhalten in kritischen Rechtssituationen“ zusammengefasst sind.

Nicht nur für jüngere Ärztinnen und Ärzte, sondern auch für die erfahrenen Kolleginnen und Kollegen stellt die Beantwortung bzw. die Lösung der aufgezeigten Aspekte nicht selten ein Problem dar, das immer häufiger ohne zeitlichen Aufschub gelöst werden muss. Aus der Sicht des Mediziners, des Medizinjuristen, des Richters, des Staatsanwalts u. a. werden die unterschiedlichen Facetten dieser vielschichtigen Problematik erläutert und praxisnah dargelegt. Nicht selten besteht Unklarheit bezüglich der Aufklärung der Eltern über Erkrankung bzw. notwendige Eingriffe bei ihren heranwachsenden Töchtern (H. Fenger, M. Entezami).

\section{(>) Dürfen die Eltern alles wissen? Grenzen der Aufklärung}

Auch die Aufklärung bei nicht einwilligungsfähigen Menschen (J. Schwarz) ist häufig Thema in Praxis und Klinik. Unklarheit besteht, ob Angehörige die Aufklärung durch den Arzt verhindern können (T. Gaibler). Es werden klare, praxisbezogene Ausführungen gemacht, die erkennen lassen, dass Angehörige ebenso wenig berufen sind, über den Kopf der Patientin hinweg zu entscheiden, wie der behandelnde Arzt. Auch das Handeln bei akuter Gefährdung der Patientin - wieviel Aufklärung? - wird unter Berücksichtigung aktueller Rechtsprechung dargelegt (G. Biermann). Nicht ausgespart bleiben darf der mehr ethisch orientierte Aspekt einer Aufklärung (J. Baltzer, L. Beck). Es wird aufgezeigt, dass auch bei der Übermittlung der Diagnose einer bösartigen Erkrankung die Wahrheit unabdingbar ist. Wahrhaftigkeit schließt Wahrheit nicht aus, sondern ist durch Empathie mit der Patientin gekennzeichnet.

Nach den Aspekten der Aufklärung gehen die folgenden Beiträge auf die Thematik der selbstverantwortlichen Patientin ein. Im umfassenden Beitrag „Patientenverfügung, Patiententestament, Palliativmedizin, Tötung auf Verlangen“ (K. Ulsenheimer) wird auf die derzeit sehr emotional geführte Diskussion mit sachlichen Argumenten eingegangen, die zeigen, dass viele diskutierte Fragen durch den Spruch des Gesetzgebers gelöst werden könnten.

\section{Viele offene inhaltliche und formale Fragen zum Patiententestament}

$\mathrm{Zu}$ bedenken ist aber, dass mit einer gesetzlichen Regelung der Problembereich in seiner Gesamtheit abschließend nicht lösbar ist. Am Beispiel einer Patientin wird die Thematik des Verzichts auf Behandlung (U. Walter) aufgegriffen und ausgeführt, welche Vorgaben notwendig sind, um ärztlicherseits bzw. medizinrechtlich den Verzicht auf Behandlung zu rechtfertigen. Das Recht auf Selbstbestimmung (M. Teske) greift die momentan kontrovers geführte Diskussion auf und formuliert aufgrund der gültigen Rechtslage eindeutige Verhaltens- und Behandlungsempfehlungen für den Arzt.

Beihilfe zum Suizid (R. Stahl) ist ebenfalls Gegenstand gegensätzlicher Diskussion. Der Beitrag gibt notwendige sachliche Informationen basierend auf der neue- ren verwaltungsgerichtlichen Judikatur. Gleiches gilt für die Thematik der Strafwürdigkeit unterlassener Hilfeleistung bei Suizidversuch (J. Schwarz). Letztlich zeigt sich, dass es auch nach höchstrichterlicher Rechtsprechung darauf ankommt, die Abwägungskriterien, die im konkreten Fall für richtig gehalten werden, zu dokumentieren, sodass die Abwägung des Arztes deutlich wird. Im Beitrag von H. Schlotz et al. wird das erschreckende Problem der verdrängten Schwangerschaft bis zur Kindstötung unter Einbeziehung möglicher Präventionsmaßnahmen dargestellt.

\section{○ Ästhetische Operationen erfordern einen besonders einfühlsamen Umgang mit der Zielvorstellung}

$\mathrm{Zu}$ den therapeutischen Maßnahmen, die nicht selten forensische Konsequenzen nach sich ziehen, zählen die ästhetischen Operationen (C. Nestle-Krämling), wenn der von der Operation erhoffte Erfolg ausbleibt. Das Problem der Leihmutterschaft bleibt nicht ausgespart (R. Felberbaum), ein Vorgehen, das im Gegensatz zu anderen Ländern in Deutschland verboten ist.

Trotz aller Vorsichtsmaßnahmen kann es zu einem Zwischenfall kommen; der „juristische Notfallkoffer“ (R.W. Bock) gibt klare Vorgaben, was zu tun ist, wenn etwas passiert ist.

Die intensive Beschäftigung mit den verschiedenen Facetten des Themas hat gezeigt, dass ein Heft für die umfangreichen Problemfelder nicht ausreichend ist, sodass gemeinsam mit dem Verlag ein zweites Heft vorgesehen ist. Während sich das vorliegende Heft überwiegend mit 
Aufklärung und Selbstverantwortlichkeit der Patientin beschäftigt, wird im Heft 1/2010 die Problematik spezieller medizinischer Eingriffe und Vorgehensweisen abgehandelt werden.

Den Autoren danken wir, dass sie trotz erheblicher beruflicher Belastung bereit waren, an diesen Themenheften mitzuarbeiten.

Wir wünschen uns zusammen mit dem Verlag, dass wir Ihnen, liebe Leserinnen und Leser, mit diesen beiden Heften Entscheidungshilfe bei Ihrer täglichen Arbeit in Praxis und Klinik vermitteln können. In akuten Sondersituationen mögen die Beiträge für Sie hilfreich sein, die richtige und zeitnahe Entscheidung zu treffen.
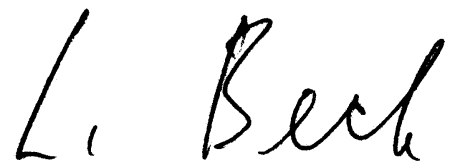

Prof. Dr. L. Beck

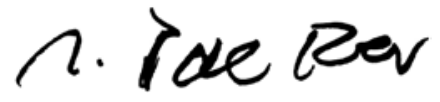

Prof. Dr. J. Baltzer

\section{Korrespondenzadressen}

\section{Prof. Dr. L. Beck}

Frauenklinik, Heinrich-Heine-Universität Moorenstr. 5, 40227 Düsseldorf

\section{Prof. Dr. J. Baltzer}

Jentgesallee 72, 47799 Krefeld prof.baltzer@the-krefelder.de

Der Gynäkologe bietet jeden Monat umfassende und aktuelle Beiträge zu interessanten Themenschwerpunkten aus allen Bereichen der Gynäkologie und Geburtshilfe.

Möchten Sie ein bereits erschienenes Heft nachbestellen? Die folgenden Ausgaben aus 2008 können Sie direkt bei unserem Kundenservice zum Preis von EUR 31,- beziehen:
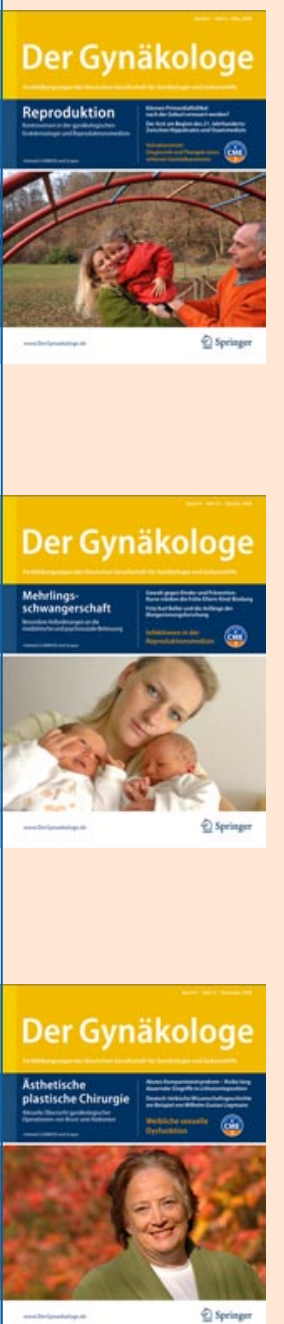

\section{8}

- 01/08 Das Umfeld moderner Geburtshilfe

- 02/08 Uterine Blutungen

- 03/08 Kontroversen in der gynäkologischen Endokrinologie und Reproduktionsmedizin

- 04/08 Nebenwirkungen onkologischer Therapien. Teil 2

- 05/08 Hysterektomie heute

- 06/08 Notfälle in der Geburtshilfe

- 07/08 Prävention gynäkologischer Krebserkrankungen

- 08/08 Palliative Medizin. Symptomkontrolle und spezielle Aspekte der Betreuung

- 09/08 Kongressheft zum 57. Kongress der DGGG

- 10/08 Besonderheiten in der Mehrlingsschwangerschaft

- 11/08 HRT, Klimakterium

- 12/08 Ästhetische plastische Chirurgie

2009

- 01/09 Habituelle Aborte

- 02/09 Risikoschwangerschaft

- 03/09 Targeted Therapy

- 04/09 Erkrankungen von Vulva und Vagina

- 05/09 40 Jahre Cardiotokographie

- 06/09 Neue Aspekte der Strahlentherapie in der Gynäkologie

- 07/09 Reproduktionsmedizin und Embryonenschutz

- 08/09 Verhalten in kritischen Rechtssituationen

- 09/09 Mammakarzinom in besonderen Situationen

- 10/09 Infektionserkrankung in Gynäkologie und Geburtshilfe

- 11/09 Prädiktive Diagnostik in der Frauenheilkunde

- 12/09 Zervixkarzinom

(Änderungen vorbehalten)

So erreichen Sie unseren Kundenservice:

Springer Medizin Verlag GmbH

Kundenservice Zeitschriften

Haberstraße 7

69126 Heidelberg

Tel. +49 $6221345-4303$

Fax: +496221 345-4229

E-Mail: subscriptions@springer.com

www.DerGynaekologe.de 